\title{
Search for Kosterlitz-Thouless transition in a triangular Ising antiferromagnet with further-neighbour ferromagnetic interactions
}

\author{
S. L. A. de Queiroz ${ }^{a \text { and Eytan } \text { Domany }^{b}}$ \\ ${ }^{a}$ Instituto de Física, Universidade Federal Fluminense, \\ Avenida Litorânea s/n, Campus da Praia Vermelha, \\ 24210-340 Niterói RJ, Brazil \\ ${ }^{b}$ Department of Physics of Complex Systems, Weizmann Institute of Science, \\ Rehovot 76100, Israel
}

(May 6, 2019)

\begin{abstract}
We investigate an antiferromagnetic triangular Ising model with anisotropic ferromagnetic interactions between next-nearest neighbours, originally proposed by Kitatani and Oguchi (J. Phys. Soc. Japan 57, 1344 (1988)). The phase diagram as a function of temperature and the ratio between first- and second- neighbour interaction strengths is thoroughly examined. We search for a Kosterlitz-Thouless transition to a state with algebraic decay of correlations, calculating the correlation lengths on strips of width up to 15 sites by transfer-matrix methods. Phenomenological renormalization, conformal invariance arguments, the Roomany-Wyld approximation and a direct analysis of the scaled mass gaps are used. Our results provide limited evidence that a Kosterlitz-Thouless phase is present. Alternative scenarios are discussed.
\end{abstract}

PACS numbers: $75.30 . \mathrm{Kz}, 75.10 . \mathrm{Hk}, 05.50+\mathrm{q}$ 


\section{INTRODUCTION}

Some spin systems display an intermediate critical phase, which is characterised by algebraic decay of correlations for a finite temperature range, and lies between the lowtemperature ordered state and the high-temperature paramagnetic regime. This was first reported by Kosterlitz and Thouless (KT) [1] in their study of the two-dimensional XY model, which exhibits long-range order only at $T=0$. The KT transition from the paramagnetic to the critical phase is expected to involve an exponential divergence of the correlation length [1].

Later it was found that some two-dimensional systems with discrete spin symmetry could accommodate critical phases as well. The first systems to be discovered in this category were the $Z(N)$ models with $N>4$ [2,3]. José et al. [2] found that, for the XY model with sixthorder anisotropy, the upper $\left(T_{1}\right)$ and lower $\left(T_{2}\right)$ limits of the KT phase are associated with the following values for the decay-of-correlations exponent $\eta(T): \eta\left(T_{1}\right)=1 / 4$ and $\eta\left(T_{2}\right)=1 / 9$. This result has been used [4] as a criterion to establish the existence and limits of a KT phase in other systems, including the one which concerns us in the present work.

Another class of spin systems with discrete symmetry displaying critical phases is obtained from models with a macroscopically degenerate ground state, by adding degeneracylifting fields or interactions. Such is the case of the nearest-neighbour Ising antiferromagnet on the triangular lattice. At $T=0$ this model has a finite entropy per spin [7], and algebraically decaying correlations [8]. The behaviour of this system in a uniform magnetic field and with second-neighbour ferromagnetic interactions was studied by Nienhuis et al. [9]. They predicted that, when the nearest neighbour interaction, $K_{n n}$, is set to $-\infty$ and the reduced field $H=B / T$ is small, the algebraic phase persists, until it is destroyed at high fields. These predictions were verified subsequently [10,11. Other predictions, involving addition of finite (reduced) second-neighbour ferromagnetic interactions, $K_{n n n}$, were made; it is expected that for large enough $K_{n n n}$ the algebraic phase exists also off the $K_{n n}=-\infty$ line (i.e., for finite $K_{n n}$ ); when $K_{n n n}$ is increased further, however, a transition to a phase with long range order is expected. This is consistent with other results [4, 12] (see below).

The triangular Ising antiferromagnet with second-neighbour ferromagnetic bonds was initially proposed to model physical adsorption of gases on graphite [13]; alternatively, it was used to describe the magnetic behaviour of $\mathrm{CsCoCl}_{3}$ and $\mathrm{CsCoBr}_{3}$ [14. The model was predicted [12] to be in the universality class of the XY model with sixth-order anisotropy [2], and thus to exhibit a KT phase. Monte Carlo results, together with the criterion of José et al., provided numerical evidence for this [4].

A phenomenological renormalisation group (PRG) analysis, however, produced clear signs of only one of the two transition temperatures associated with the KT phase [15. This discrepancy was ascribed to the small strip widths used, namely 3 and 6 . In order to be able to analyse larger strip widths, Kitatani and Oguchi [5] introduced a slightly different model which incorporates only four of the six second-neighbour interactions per site. Each of the three second-neighbour sublattices then forms a square lattice, instead of the original triangular one. This introduces spatial anisotropy, with consequences which we shall discuss in what follows. In the original work of Ref. [5], the underlying hypothesis is that, since the two-dimensional character of the intrasublattice links was preserved, the qualitative behaviour ought to be in the same universality class of the model with all six 
interactions per site. Those authors managed to study strips of width up to 12 sites with periodic boundary conditions (to which the Roomany-Wyld approximation [16] for the $\beta$ function [17] was applied), and 17 with helical boundary conditions (for which the spinspin correlation functions were calculated directly and fitted to the criterion of José et al.). While results from the former approach were deemed inconclusive, the authors pointed out that the latter provided evidence of a KT phase. Subsequent Monte Carlo work was found to be consistent with this [6] by means of a finite-size scaling and renormalisation-group analysis of the simulation data, partly with help from the criterion of José et al.. Very recently, free energy and magnetisation were calculated for this model [18] by a combination of transfer-metrix and mean-field ideas. The authors find an intermediate, incommensurate phase. The KT transition, in this interpretation, would be commensurate-incommensurate in character. An exact analysis of the ground-state of the model with second-neigbour bonds along only one direction also shows evidence of a critical phase [19]; when interactions along a second direction are included, so as to make the model identical with the present one, an exact treatment is no longer possible, even for the ground state. However, Monte-Carlo and transfer matrix data also point towards a commensurate-incommensurate transition at zero temperature (i.e. when the first- neighbour couplings are set at $-\infty$ ) in this case, as the strength of second-neigbour bonds varies [20]. The latter results are not to be directly compared to ours, as they pertain to the interacting domain-wall transitions which take place between ground-state configurations.

Here we study the model proposed by Kitatani and Oguchi [5] on strips of width 3, 6, 9, 12 and 15 with periodic boundary conditions. The interpretation of PRG results in searching for a KT phase is discussed, and these are shown to be of limited usefulness when considered on their own. The Roomany-Wyld (RW) approximation [16 for the $\beta$ function [17] is implemented, for comparison with the corresponding results of Kitatani and Oguchi; a direct analysis of the ratio between the correlation lengths on strips of different widths (which, for quantum systems, correspond to the scaled mass gaps) [16,21,22] is given as well. We discuss the bearing of conformal invariance theory on the comparison of our results to previous ones. Finally we try to provide a consistent framework for our findings.

\section{PHASE DIAGRAM; SPECIFIC HEATS}

The model is characterised by the nearest-neighbour interaction $J_{n n}<0$ and the secondneighbour coupling $J_{n n n}>0$, or alternatively by the ratios of these to the temperature, respectively $K_{n n}=J_{n n} / T$ and $K_{n n n}=J_{n n n} / T$. A useful parameter is the ratio $R \equiv J_{n n} / J_{n n n}$. Kitatani and Oguchi [5] restricted themselves to $R=-2.0$, whereas Miyashita et al [6] considered both $R=-2.0$ and $R=-5.0$. We span the region $-20.0 \leq R \leq 0.0$.

A phase diagram which, though not completely consistent with all our findings, appears to be quite plausible, is presented in Fig. 1. Near the origin there is a high-temperature paramagnetic phase. Above the solid line we predict existence of a low-temperature phase with long-range order. The shaded region in between is our candidate for a critical (or massless) KT phase. Fixed- $R$ lines, along which detailed studies were carried out, are also indicated.

An examination of specific heats along lines of fixed $R$ unveils a two-peak structure. This is especially apparent for $R=-2.0$, as shown in Fig. 2 for $L=3, \ldots, 15$, for comparison 
with Fig. 3 of Ref. [5], where $L=9$ is the largest width used. However, when other values of $R<-2.0$ are investigated, the high-temperature peaks are displaced to ever higher temperatures and turn much broader, though those at the low end remain sharp and essentially unmoved. Fig. 3 illustrates this, with data for $L=15$ and $R=-2.0,-5.0$ and -20.0 . The specific heat peaks apparently do not diverge with increasing $L$. Hence their positions do not identify transition points. The double-peak structure can be used only as a qualitative indicator of resemblance to $Z(N)$ models with $N>4$, which also exhibit such structure, and for which an intermediate massless phase is known to exist [2, 3]. We shall not return to specific heats; instead, our work is focused mainly on correlation lengths and quantities derived therefrom, which will prove to be a safer ground for analysis.

\section{PHENOMENOLOGICAL RENORMALIZATION}

We begin by performing standard phenomenological renormalisation. The PRG procedure consists of searching, for fixed $R$, for the fixed point $\left\{K_{n n}^{*}, K_{n n n}^{*}\right\}$ of the implicit recursion relation

$$
\frac{\xi_{L^{\prime}}\left(K_{n n}^{\prime}, K_{n n n}^{\prime}\right)}{L^{\prime}}=\frac{\xi_{L}\left(K_{n n}, K_{n n n}\right)}{L}
$$

where $\xi_{L}$ is the correlation length along a strip of width $L$. Usually best results are obtained for $L^{\prime} / L \rightarrow 1$; as in the present case sublattice symmetry demands that these be multiples of 3, we use $L^{\prime}=L-3$. The correlation length is given by $\xi_{L}=1 /\left(\zeta \log \lambda_{1} /\left|\lambda_{2}\right|\right)$, where the geometric factor $\zeta$ is [10,11] $(2 / \sqrt{3})$ for $K_{n n} \neq 0$ and $\sqrt{2}$ for $K_{n n}=0 ; \lambda_{1,2}$ are the two largest eigenvalues (in absolute value) of the $2^{L} \times 2^{L}$ transfer matrix between adjacent columns of a strip of width $L$. As $\lambda_{1}$ is related to the strip free energy, it is always positive, while $\lambda_{2}$ in the present case is one of a pair of complex conjugate eigenvalues with arguments $\pm 2 \pi / 3$ (owing to the three-sublattice structure). If all six second-neighbour bonds were present [15], the transfer matrix would have to take into account adjacent pairs of columns, raising its dimension to $2^{2 L} \times 2^{2 L}$. Omission of two out of the six next-nearest neighbour couplings is thus technically worthwhile. One should, however, bear in mind the fact that, by doing this, the six-fold symmetry of the problem has been broken. Even though the model still has a six-fold degenerate ground state, the energies associated with domain walls between these phases depend now on the orientation of the walls, and one has to work out the symmetry of this new problem. It is quite clear that the criterion $\eta=1 / 9$ of José et al [2] to locate the low-temperature transition to a six-fold symmetric ordered phase should not be naively used here.

For a system with a KT phase, one could expect Eq. 1 to exhibit a line of fixed points along a finite temperature interval, corresponding to the extent of the intermediate phase [17]. Finite systems should display a precursor for such behaviour, such as having more than one solution to Eq. 1 at nearby points, in the region where a KT phase is expected. This does not happen in the present case, although the direct analysis of the scaled mass gaps (to be discussed below) shows that there are extensive temperature ranges along which other indicators of a massless phase occur. For each given $R$ and $L^{\prime} / L$, we find only one temperature as a fixed point of Eq. 1. In Fig. 14 we display the approximate critical lines given by the fixed points of Eq. 1 on the $\left(K_{n n}, K_{n n n}\right)$ plane, for the several 
values of $L^{\prime} / L$. Points on the extrapolated curve are obtained by keeping $R$ constant and plotting the respective fixed-point couplings (e.g. $K_{n n n}$ ) for $L^{\prime} / L=6 / 9,9 / 12$ and 12/15 against $L^{-\psi}$, where $\psi$ is chosen to give the best straight-line fit through the three points, and is an estimate of the corrections-to-scaling exponent [23]. We find that $\psi$ falls roughly in the range $\sim 1-3$ wherever such extrapolation is applicable (see below).

We have also applied domain-wall renormalisation group ideas 24 by reversing the sign of the interactions that cross a line along the strip, which amounts to establishing antiperiodic boundary conditions [25]. The difference between the corresponding free energy and that for a strip with standard periodic boundary conditions gives the domain-wall free energy, which vanishes at criticality [26] and is the quantity to be scaled. Again, only one fixed point was found for each $R$. The extrapolated phase diagram thus obtained (not shown here) is roughly the same as that coming from correlation-length scaling. Consequently, we did not pursue this line further. It is worth noting, however, that the finite- $L$ data approach the extrapolation from the high-temperature side while those from standard PRG (see Fig. (4) come from below. This is to be expected from well-known duality relations between domain-wall energy and inverse correlation length [26].

For isotropic systems, conformal invariance [27] allows one to extract additional information from strip scaling, via the relationship between correlation-length amplitudes on a strip of width $L$ at criticality and $\eta$, the decay-of-correlations exponent :

$$
\eta=L / \pi \xi\left(T_{c}\right)
$$

Here, anisotropy is introduced by the missing second-neighbour bonds. Critical correlations are expected to decay with different prefactors in different directions, though with the same exponent. In some cases this can be explicitly dealt with [28 30], leading to a modified form for Eq. 2 which involves knowledge of correlation lengths along two different directions [28]. Unfortunately this it out of reach in the present model, whose very introduction was in order to enable one to build large- $L$ transfer matrices along the specific direction where the bonds are missing. For $K_{n n}=0$ or $K_{n n n}=0$, isotropy is restored and Eq. 2 is valid, with results to be described below.

Referring to Fig. 4, the following comments are in order:

(1) On the vertical axis $\left(K_{n n}=0\right)$ one has three superimposed, disjoint, square lattices; thus the $L$ arising in the conformal-invariance expression for $\eta$ is one-third of the number of sites in the column. The corresponding critical quantities, extrapolated as above, are (exact values quoted in parenthesis): $K_{n n n}(0)=0.441 \pm 0.001(0.4407 \ldots) ; y_{T}=1 / \nu=1.00 \pm 0.01(1)$; $\eta=0.250 \pm 0.001(1 / 4)$.

(2) The extrapolated boundary leaves the vertical axis tangentially with a crossover exponent $\phi \simeq 1.55$, where $\left|K_{n n}\right| \sim\left(K_{n n n}(0)-K_{n n n}\right)^{\phi}$. This can be compared with the exact $\phi=7 / 4$ obtained by Slotte and Hemmer 31] for the corresponding model with antiferromagnetic second-neighbour interactions.

(3) In the region between $R \simeq-0.01$ and $R \simeq-0.3$ the approximate critical lines become very close and cross each other twice. Thus, an extrapolation similar to that of Ref. [23] is not feasible.

(4) As $R \rightarrow-\infty$ the critical lines become horizontal; the extrapolated value is $K_{n n n}=$ $0.313 \pm 0.001$. Already at $R=-2.0$ one is within less than $1 \%$ of that. Qualitatively, as $\left|K_{n n}\right|$ grows the only ratio that matters is that between the two finite energies present, 
namely $J_{n n n}$ and $T$. Such quantity is analogous to the magnetic field-to-temperature ratio in the zero-temperature nearest-neighbour triangular antiferromagnet [10,11]. In order to check our results against those of Refs. [10,11], we set $K_{n n n}=0$ and make $\left|K_{n n}\right|$ large. This must coincide with their zero-field limit. Indeed we obtain $\eta=0.4815,0.4909,0.4947$, 0.4965 respectively for $L=6,9,12$ and 15, results in excellent visual agreement with Fig. 2 of Ref. [10] and converging towards the exact value [8] $\eta=1 / 2$.

The question remains of the interpretation of the critical line given by PRG in the context of KT transitions, where two phase changes are expected to occur. Following previous work [5].6], we try to fit our results to the criterion of José et al. [2]. To this end we examine the exponent $\eta$ on the critical line. Bearing in mind the above remarks concerning anisotropy, the quantity $L / \pi \xi\left(T_{c}\right)$ does not in general correspond to $\eta$; however, for large $\left|K_{n n}\right|$ and finite $K_{n n n}$ one has essentially a triangular antiferromagnet with weak anisotropy induced by the square-lattice ferromagnetic bonds. In this region, corresponding to the horizontal section of the critical line in Fig. 14, Eq. 2 is expected to become asymptotically true. Fig. 5 shows $L / \pi \xi\left(T_{c}\right)$ as a function of $K_{n n}$. It can be seen that for large $\left|K_{n n}\right|, L / \pi \xi\left(T_{c}\right)$ becomes constant and extrapolates to 0.25 from below. On the basis of this, one would tend to state that the critical line of PRG marks the high temperature boundary $T_{1}$ of the KT phase, at least in the region where the antiferromagnetic coupling is strong. This, however, is inconsistent with previous findings [5,6] (see below). Further, inspection of the $\xi_{L} / L$ vs. $T$ curves for large $|R|$ shows no evidence of non-monotonic behaviour; in particular, no trend is found towards a second crossing close to temperatures where conformal invariance would give $\eta$ near 1/9. An example is shown in Fig. 6; for $R=-5.0$, at the couplings for which $L / \pi \xi=1 / 9$ we do not see any tendency for the different- $L$ lines to cross, or even to approach one another. We then consider other quantities, in order to estimate bounds for a possible KT phase.

\section{ROOMANY-WYLD APPROXIMATION}

To make contact with previous results, we investigate the Roomany-Wyld (RW) approximation [16] for the $\beta$ function [17], as done in Ref. [5] for $R=-2.0$. This is given by

$$
\beta_{L L^{\prime}}^{R W}(T)=\frac{\log \left(\xi_{L} / \xi_{L^{\prime}}\right) / \log \left(L / L^{\prime}\right)-1}{\left\{\xi_{L}^{\prime} \xi_{L^{\prime}}^{\prime} /\left(\xi_{L} \xi_{L^{\prime}}\right)\right\}^{1 / 2}}
$$

where $\xi^{\prime}$ denotes a derivative of $\xi$ with respect to temperature. Note that $\beta_{L L^{\prime}}^{R W}=0$ at the fixed point of the PRG transformation (Eq. 1), and one would expect it to remain at zero for an extended temperature interval corresponding to a KT phase. This would be in contrast with the monotonic behaviour exhibited in systems with an ordinary transition [17]. In Fig. 7 we display $\beta_{L L^{\prime}}^{R W}$ against temperature for $R=-2.0$ and $L^{\prime} / L=3 / 6,6 / 9,9 / 12$ (already obtained in Ref. [5]) and 12/15. Although the $3 / 6$ curve is almost featureless, a trend arises towards flattening as $L$ increases, especially when the 12/15 data are considered. The range of temperatures for which one would, from visual inspection, expect the $L \rightarrow \infty$ curves to touch the axis, is in broad agreement with the approximate boundaries of the KT phase as obtained by previous authors [5, [6]. 
Fig. 8 shows $\beta_{L L^{\prime}}^{R W}$ against temperature for the largest strip width $L=15\left(L^{\prime}=12\right)$ and several values of $R$. It becomes apparent that the trend towards flattening noticed earlier turns even weaker, on either side of $R=-2.0$. As the RW approximation produces clear-cut plateaus in other cases where a critical phase is present (see e.g., Fig.6 of ref. [16]), one is led to concur with Kitatani and Oguchi's conclusion, by deeming the present results inconclusive for general $R$.

\section{SCALED MASS GAPS}

We then consider the ratio

$$
\mathcal{Q} \equiv\left(\xi_{L}(T) / L\right) /\left(\xi_{L^{\prime}}(T) / L^{\prime}\right)
$$

This quantity was introduced in the study of the scaled mass gaps of quantum systems [16, 21], and was used in Ref. [22] in the study of anisotropic Ising triangular antiferromagnets in an external field, which exhibit features similar to those under examination here. At the fixed point of PRG, $\mathcal{Q}=1$ and one expects it to remain close to this value for an extended temperature interval if a critical phase is present. This is numerically verified to a good extent in several cases 16,21,22. In Figures 9 and 10 we show $\mathcal{Q}$ respectively for $R=-2.0$ and -5.0 , the same values considered in Ref. [6], and several pairs of strip widths. Figure 11 displays plots of $\mathcal{Q}$ against temperature for $L=15$ and $L^{\prime}=12$, and $R=-0.5$, $-2.0,-5.0$ and -20.0 .

Note that the fractional deviation of $\mathcal{Q}$ from 1 is much smaller than the corresponding shift of $\beta_{L L^{\prime}}^{R W}$ from zero in Fig. 8. As $|R|$ grows, the extent of the flattening region increases. For $R=-20.0$ and $L=15, \mathcal{Q}$ rises quite steeply from 0.95 at $T / J_{n n n}=2.4$ to 1.0 at $T / J_{n n n} \simeq 2.96$, the fixed point of PRG, and then remains below 1.005 up to $T / J_{n n n}=10.0$.

The data from $\mathcal{Q}$ indicate that, at least for $R<-2.0$, there is a signature consistent with the presence of a KT phase at temperatures above that of the fixed point of PRG. This would support the results of Refs. [5, 6]. Visual extrapolation of the temperature range for which $\mathcal{Q}$ approaches 1 for large $L$ would give the upper limits $T_{1} / J_{n n n} \simeq 4.0$ and $\simeq 5.2$ respectively for $R=-2.0$ and -5.0 . These compare well with the corresponding Monte Carlo results [6] $T_{1} / J_{n n n} \simeq 3.8$ and $\simeq 5.0$. On the other hand, our evidence strongly suggests that the lower limit $T_{2}$ must be the fixed point of PRG, whose extrapolated location is at $T_{2} / J_{n n n} \simeq 3.19$, both for $R=-2.0$ and -5.0 . This value is definitely higher than those quoted by Miyashita et al, resp. $\sim 2.6$ and $\sim 2.75$.

For small $|R|$ the signature referred to above tends to disappear. If the KT phase is present at all, it must vanish as $R \rightarrow 0$, since at $R=0$ one has a simple structure of three independent, superimposed, ferromagnetic square lattices. In this context, one cannot rule out the possible existence of a multicritical point at some $R_{t} \neq 0$, such that for $|R|<\left|R_{t}\right|$ there would be only an ordinary transition. However, we have not investigated this aspect in detail.

The trends arising from the examination of $\beta^{R W}$ and, especially, $\mathcal{Q}$ are difficult to reconcile with the results for $\eta$ coming from conformal invariance (though the latter are, admittedly, valid only for large $|R|$ when anisotropy becomes weak). One would expect $\eta \simeq 1 / 4$ at the high-temperature end of the massless phase, and $\eta<1 / 4$ at low temperatures still within the critical phase. This, however, is not seen in our results (recall, e.g. Fig. 6). 


\section{DISCUSSION AND CONCLUSIONS}

We have analysed four quantities for a wide range of values of the first-to-secondneighbour interaction ratio $R$ in the Kitatani-Oguchi model: $(a)$ the location of the fixed point of PRG; $(b)$ the critical exponent $\eta$, as given by conformal invariance; $(c)$ the $\beta$ function in the Roomany-Wyld approximation; $(d)$ the scaled mass gap $\mathcal{Q}$. Overall, one can say that $(c)$ does not provide conclusive evidence as regards the existence of a KT phase. On the other hand, the positions of the seemingly clear-cut plateaus arising from $(d)$, above the fixed point of PRG given by $(a)$, do indicate existence of a massless phase; however the values of $\eta$ obtained in this region, from $(b)$, are inconsistent with the standard expectations.

One possibility is that the model of Kitatani and Oguchi does not display a KT phase at all, owing to the fact (discussed above) that it is not six-fold symmetric. In this scenario, the transformation of the second-neighbour sublattices from triangular to square via the deletion of the appropriate bonds would be relevant in the renormalisation-group sense. For example, the work of José et al [2] indicates that, for the $X Y$ model with fourth-class anisotropy one has only one transition, with $\eta=1 / 4$.

Although this is an appealing conjecture, we feel that our data from $(d)$ for large $|R|$ definitely show a trend towards the onset of a critical phase. Based on this, we propose the approximate phase diagram shown in Fig. 1, where the boundaries of the shaded area are obtained from extrapolation of the flat sections of the scaled mass gap diagrams.

It is worth recalling that other generalisations of the triangular Ising antiferromagnet also display critical phases, without obeying the criterion of José et al. We refer specifically to the model with second-neighbour ferromagnetic bonds along only one lattice direction [19], and to one with continuous degrees of freedom superimposed on the original Ising ones [32. For the present model, we recall the zero-temperature transitions between domain states [20], and single out the Monte-Carlo renormalisation group results of Miyashita et al, summarised in Fig. 6 of Ref. [6], which do point towards the existence of a critical phase, and were obtained without recourse to the criterion of José et al. It would seem that this latter criterion is valid only for the model for which it was originally formulated [2] and its closest relatives [4] with full six-fold symmetry.

\section{ACKNOWLEDGMENTS}

The authors would like to thank the Department of Theoretical Physics at Oxford, where this work was initiated, for the hospitality. SLAdQ thanks D. P. Landau, C. Vanderzande. M. Henkel and J. Cardy for useful conversations, and Departamento de Física, PUC/RJ for use of their computational facilities. Research of SLAdQ is partially supported by the Brazilian agencies Ministério da Ciência e Tecnologia, Conselho Nacional de Desenvolvimento Científico e Tecnológico and Coordenação de Aperfeiçoamento de Pessoal de Ensino Superior. ED acknowledges partial support of the US-Israel Binational Science Foundation $(\mathrm{BSF})$. 


\section{REFERENCES}

* Electronic address: sldq@if.uff.br

[1] J.M. Kosterlitz and D.J. Thouless, J. Phys. C 6, 1181 (1973).

[2] J.V. José, L.P. Kadanoff, S. Kirkpatrick and D.R. Nelson, Phys. Rev. B 16, 1217 (1977).

[3] S. Elitzur, R.B. Pearson and J. Shigemitsu, Phys. Rev. D 19, 3698 (1979).

[4] D.P. Landau, Phys. Rev. B 27, 5604 (1983).

[5] H. Kitatani and T. Oguchi, J. Phys. Soc. Japan 57, 1344 (1988).

[6] S. Miyashita, H. Kitatani and Y. Kanada, J. Phys. Soc. Japan 60, 1523 (1991).

[7] R.M.F. Houtappel, Physica 16, 425 (1950).

[8] J. Stephenson, J. Math. Phys. A 11, 413 (1970).

[9] B. Nienhuis, H.J. Hilhorst and H.W.J. Blöte, J. Phys. A 17, 3559 (1984).

[10] H.W.J. Blöte, M.P. Nightingale, X.N. Wu and A. Hoogland, Phys. Rev. B 43, 8751 (1991).

[11] H.W.J. Blöte and M.P. Nightingale, Phys. Rev. B 47, 15046 (1993).

[12] E. Domany, M. Schick, J.S. Walker and R.B. Griffiths, Phys. Rev. B 18, 2209 (1978).

[13] C.E. Campbell and M. Schick, Phys. Rev. A 5, 1919 (1972).

[14] M. Mekata, J. Phys. Soc. Japan 42, 76 (1977).

[15] Y. Saito and G. Tabe, J. Phys. Soc. Japan 54, 2955 (1985).

[16] H.H. Roomany and H.W. Wyld, Phys. Rev. D 15, 3341 (1980).

[17] M. N. Barber, in Phase Transitions and Critical Phenomena, edited by C. Domb and J.L. Lebowitz (Academic, New York, 1983), vol. 8 .

[18] P. Pajersky and A. Surda, J. Stat. Phys. 76, 1467 (1994).

[19] J.D. Noh and D. Kim, Phys. Rev E 49, 1943 (1994).

[20] J.D. Noh and D. Kim, Phys. Rev E 51, 226 (1995).

[21] C.J. Hamer and M.N. Barber, J. Phys. A 14, 259 (1981).

[22] B. Schaub and E. Domany, Phys. Rev B 28, 2897 (1983); E. Domany and B. Schaub, Phys. Rev B 29, 4095 (1984).

[23] B. Derrida and D. Stauffer, J. Physique 46, 1623 (1985) .

[24] W.L. McMillan, Phys. Rev B 29, 4026 (1984) .

[25] J. L. Cardy, J. Phys. A 17, L961 (1984) .

[26] P.G. Watson, in Phase Transitions and Critical Phenomena, edited by C. Domb and M.S. Green (Academic, New York, 1972), vol. 2 .

[27] J. L. Cardy, in Phase Transitions and Critical Phenomena, edited by C. Domb and J.L. Lebowitz (Academic, New York, 1987), vol. 11 .

[28] M.P. Nightingale and H.W.J. Blöte, J. Phys. A 16, L657 (1983) .

[29] M.N. Barber, I. Peschel and P.A. Pearce, J. Stat. Phys. 37, 497 (1984) .

[30] D. Kim and P. A. Pearce, J. Phys. A 20, L451 (1987) .

[31] P.A. Slotte and P.C. Hemmer,J. Phys. C 17, 4645 (1984) .

[32] P. Chandra, P. Coleman and B. Ioffe, Phys. Rev B 49, 12897 (1994). 


\section{FIGURES}

FIG. 1. Qualitative phase diagram. Heavy line is extrapolated from PRG (see Fig. 4). Shaded area gives massless phase, as obtained from scaled mass gaps. Constant- $R$ lines are shown, along which detailed studies were carried out. $T$ marks (conjectured) multicritical point (see text).

FIG. 2. Specific heat against temperature for $R=-2.0$. Bottom to top: $L=3,6,9,12,15$.

FIG. 3. Specific heat against temperature for $L=15$ and $R=-2.0,-5.0$ and -20.0 .

FIG. 4. Phase diagram in $K_{n n}-K_{n n n}$ space, as given by the fixed points of PRG. The heavy line is an extrapolation from the 6/9, 9/12 and 12/15 curves. Some constant- $R$ lines are shown.

FIG. 5. $L / \pi \xi_{L}$ along the critical curves of PRG. The heavy line is an extrapolation from the $6 / 9,9 / 12$ and $12 / 15$ curves.

FIG. 6. $L / \pi \xi_{L}$ against temperature for $R=-5.0$. Horizontal lines at $1 / 9$ and $1 / 4$ mark the values of $\eta$ at the boundaries of the KT phase for the XY model with sixth-order anisotropy.

FIG. 7. Roomany-Wyld approximant $\beta_{L L^{\prime}}^{R W}$ against temperature for $R=-2.0$, and $L / L^{\prime}=3 / 6$, $6 / 9,9 / 12$ and $12 / 15$.

FIG. 8. Roomany-Wyld approximant $\beta_{L L^{\prime}}^{R W}$ against temperature for $L / L^{\prime}=12 / 15$ and $R=$ $-0.5,-2.0,-5.0$ and -20.0 .

FIG. 9. $\mathcal{Q} \equiv\left(\xi_{L}(T) / L\right) /\left(\xi_{L^{\prime}}(T) / L^{\prime}\right)$ against temperature for $R=-2.0$ and $L / L^{\prime}=3 / 6,6 / 9$, $9 / 12$ and $12 / 15$.

FIG. 10. $\mathcal{Q} \equiv\left(\xi_{L}(T) / L\right) /\left(\xi_{L^{\prime}}(T) / L^{\prime}\right)$ against temperature for $R=-5.0$ and $L / L^{\prime}=3 / 6,6 / 9$, 9/12 and $12 / 15$.

FIG. 11. $\mathcal{Q} \equiv\left(\xi_{L}(T) / L\right) /\left(\xi_{L^{\prime}}(T) / L^{\prime}\right)$ against temperature for $L / L^{\prime}=12 / 15$ and $R=-0.5$, $-2.0,-5.0$ and -20.0 . 


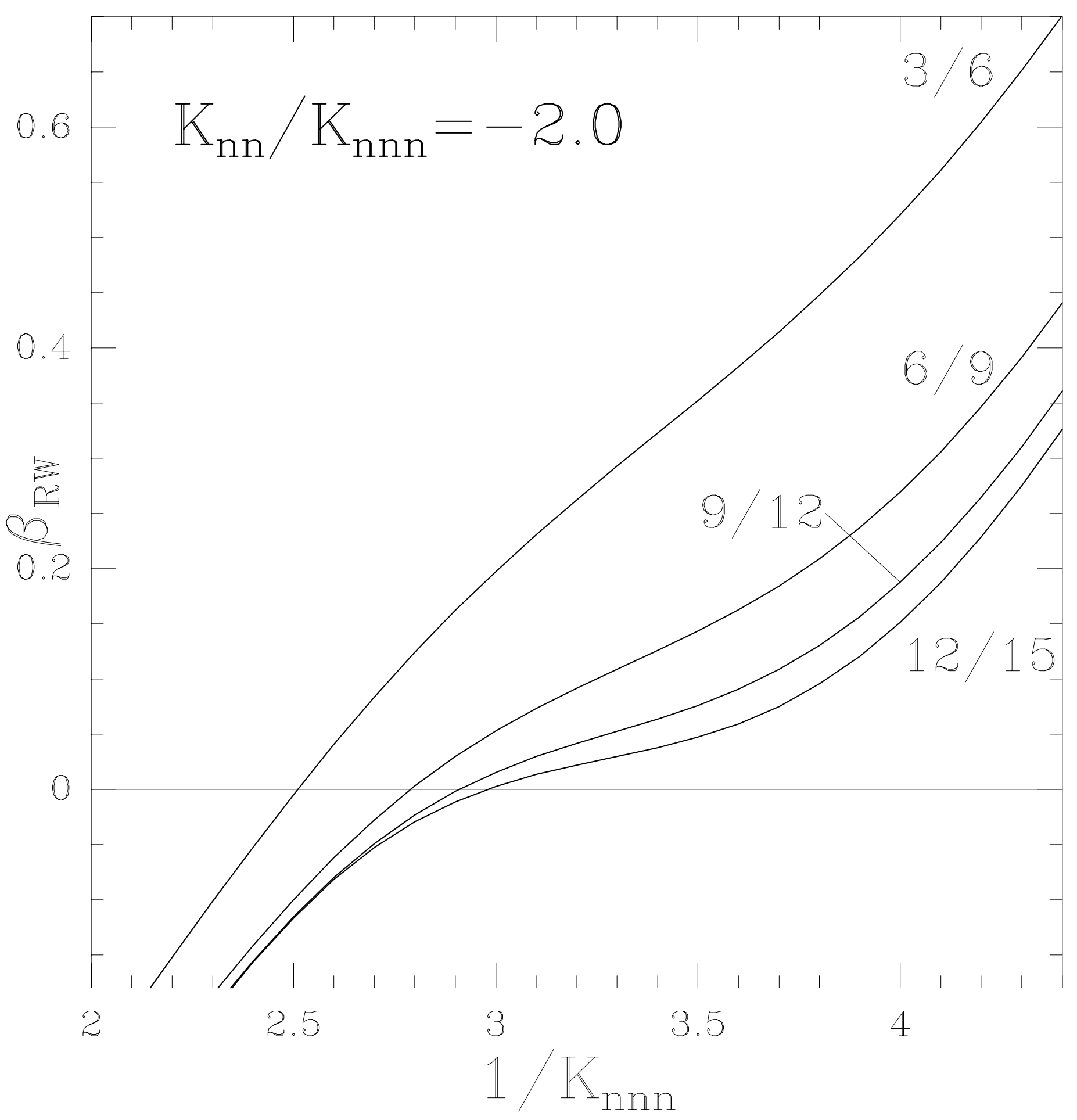




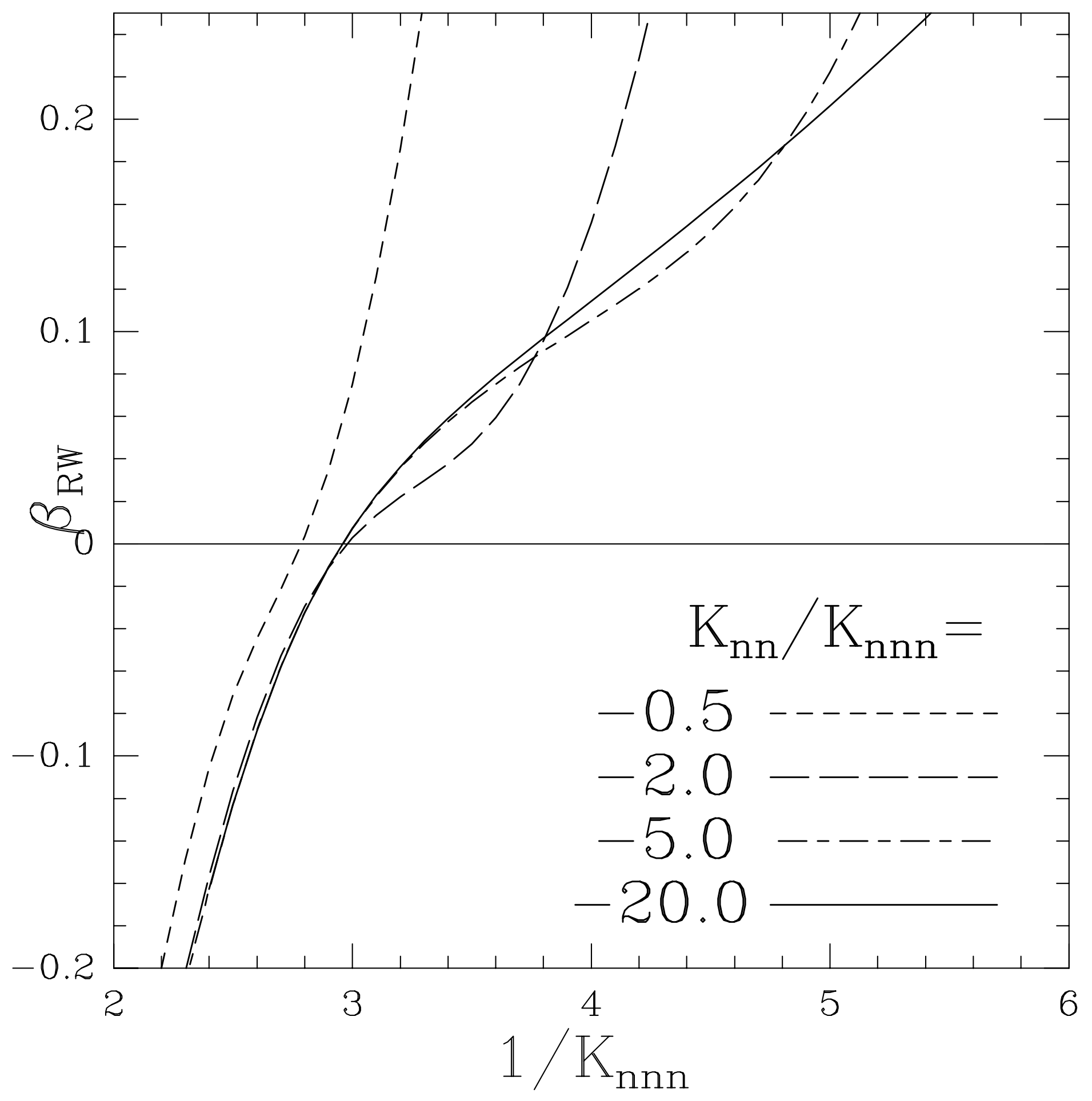




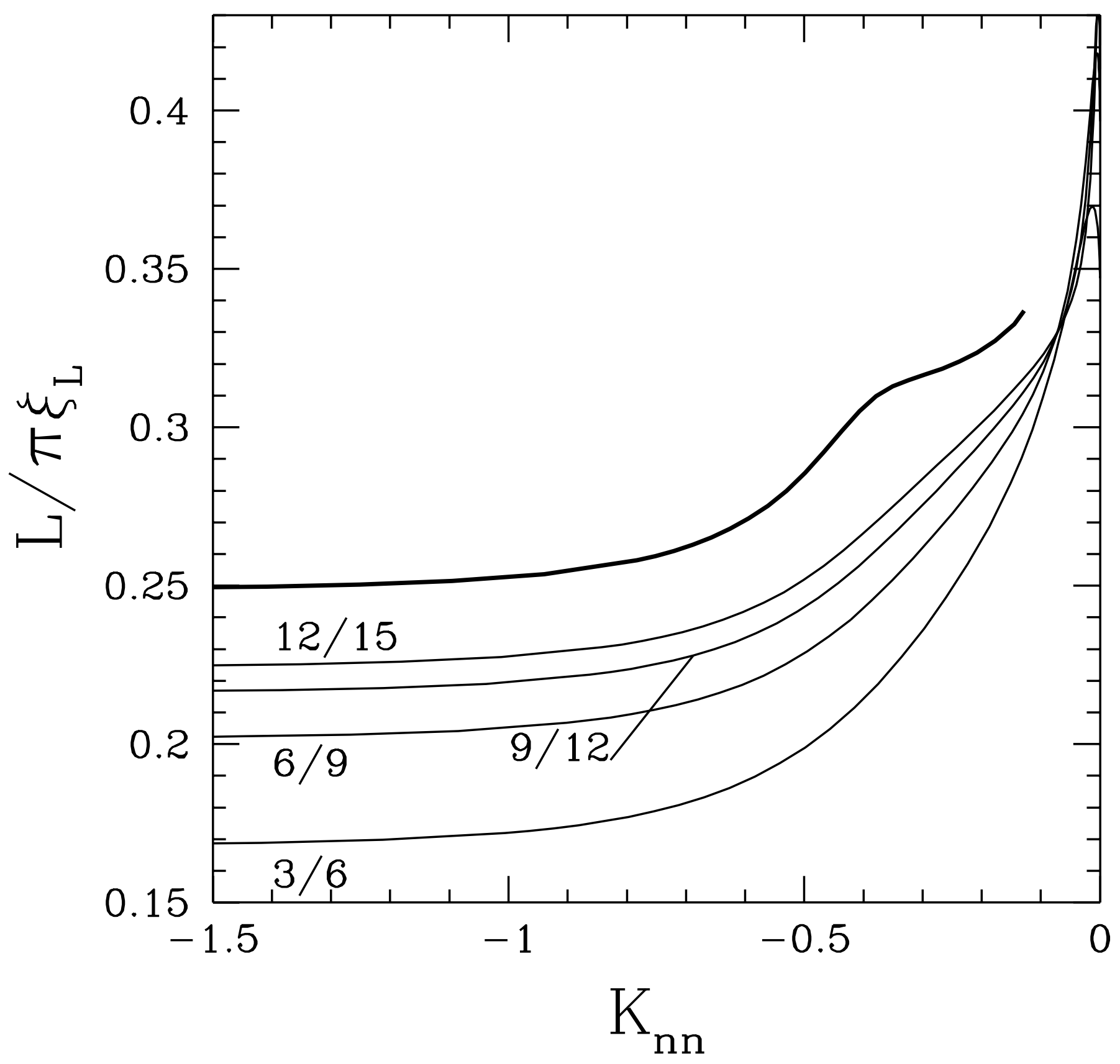




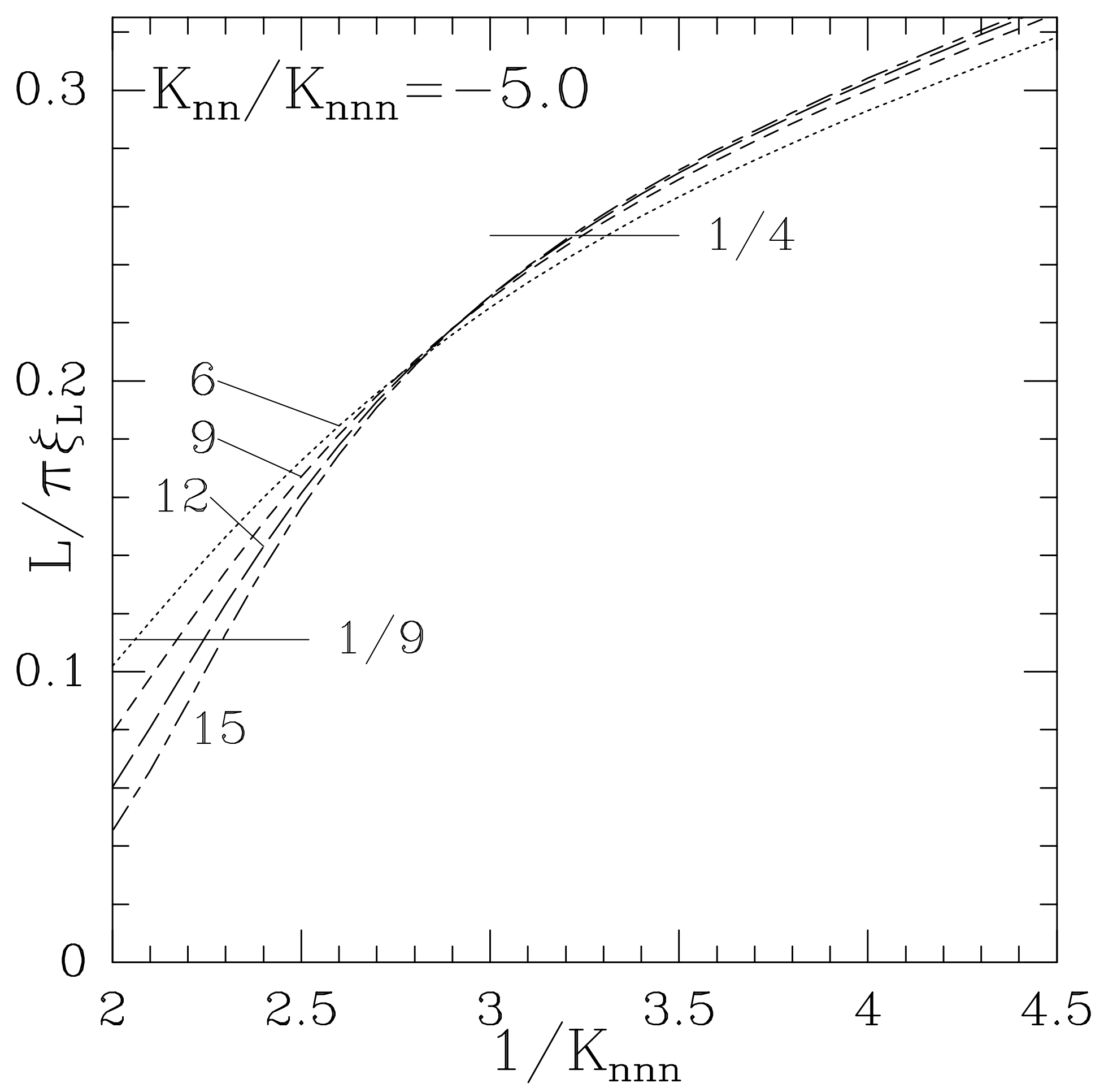




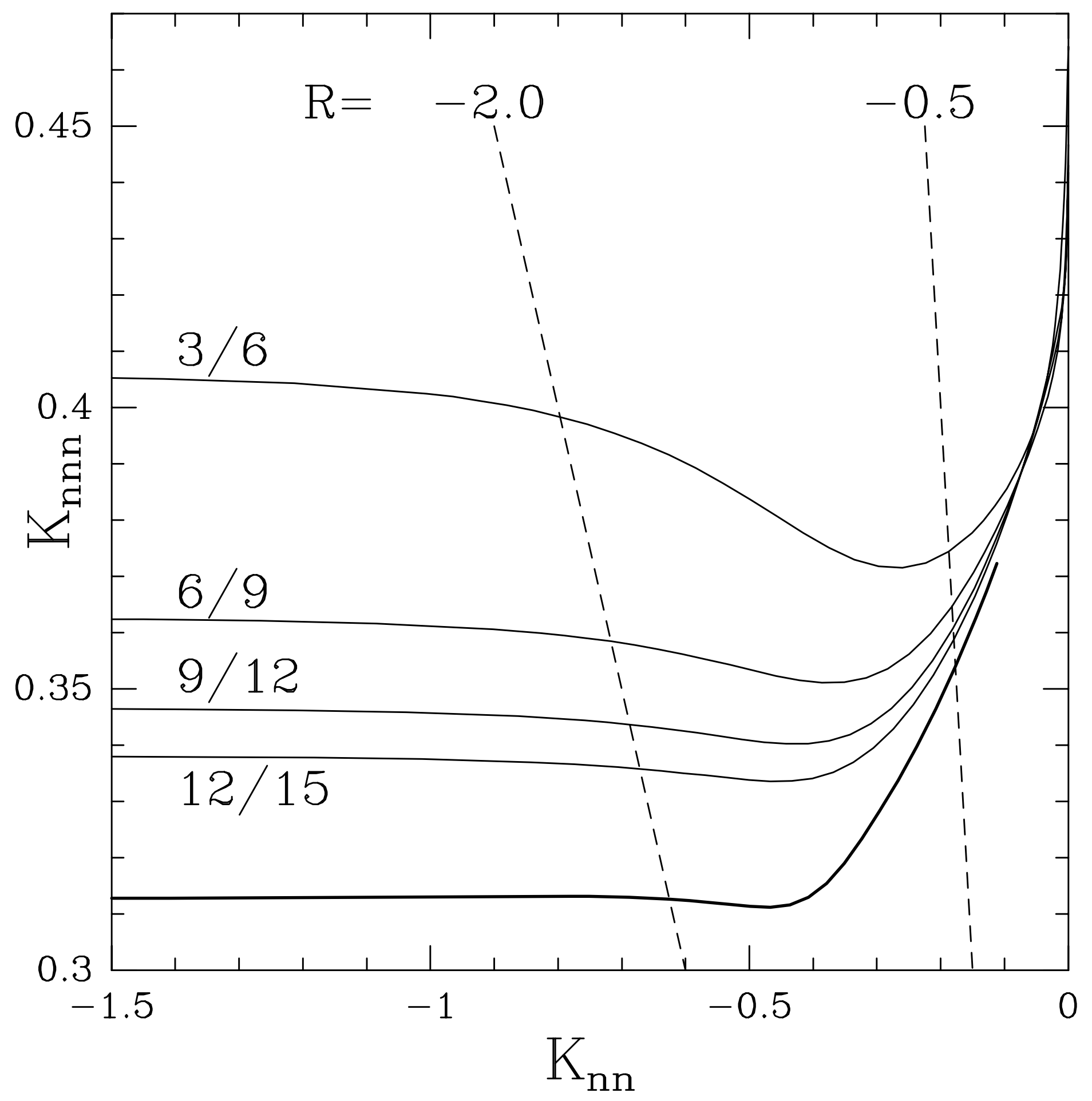




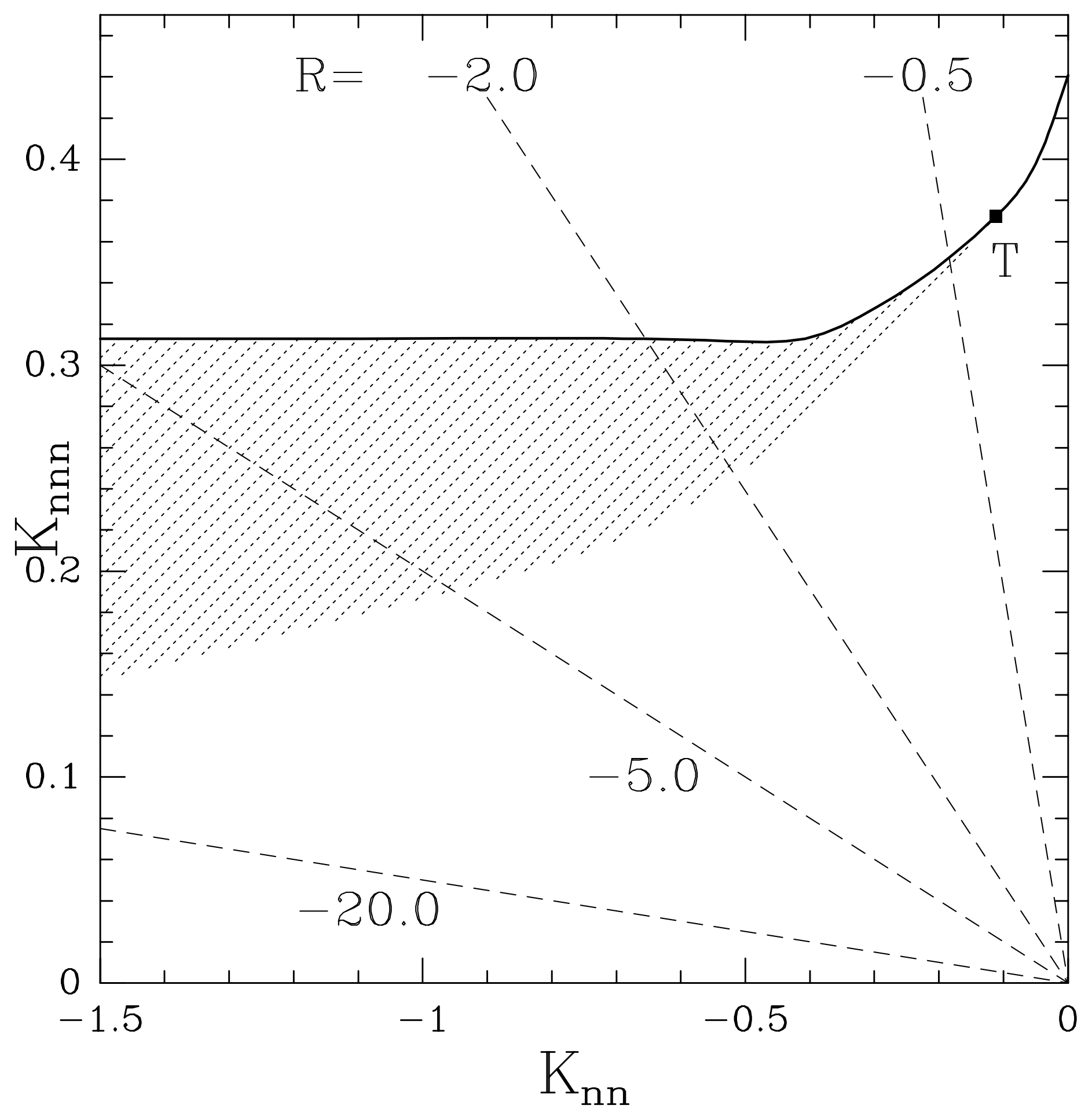




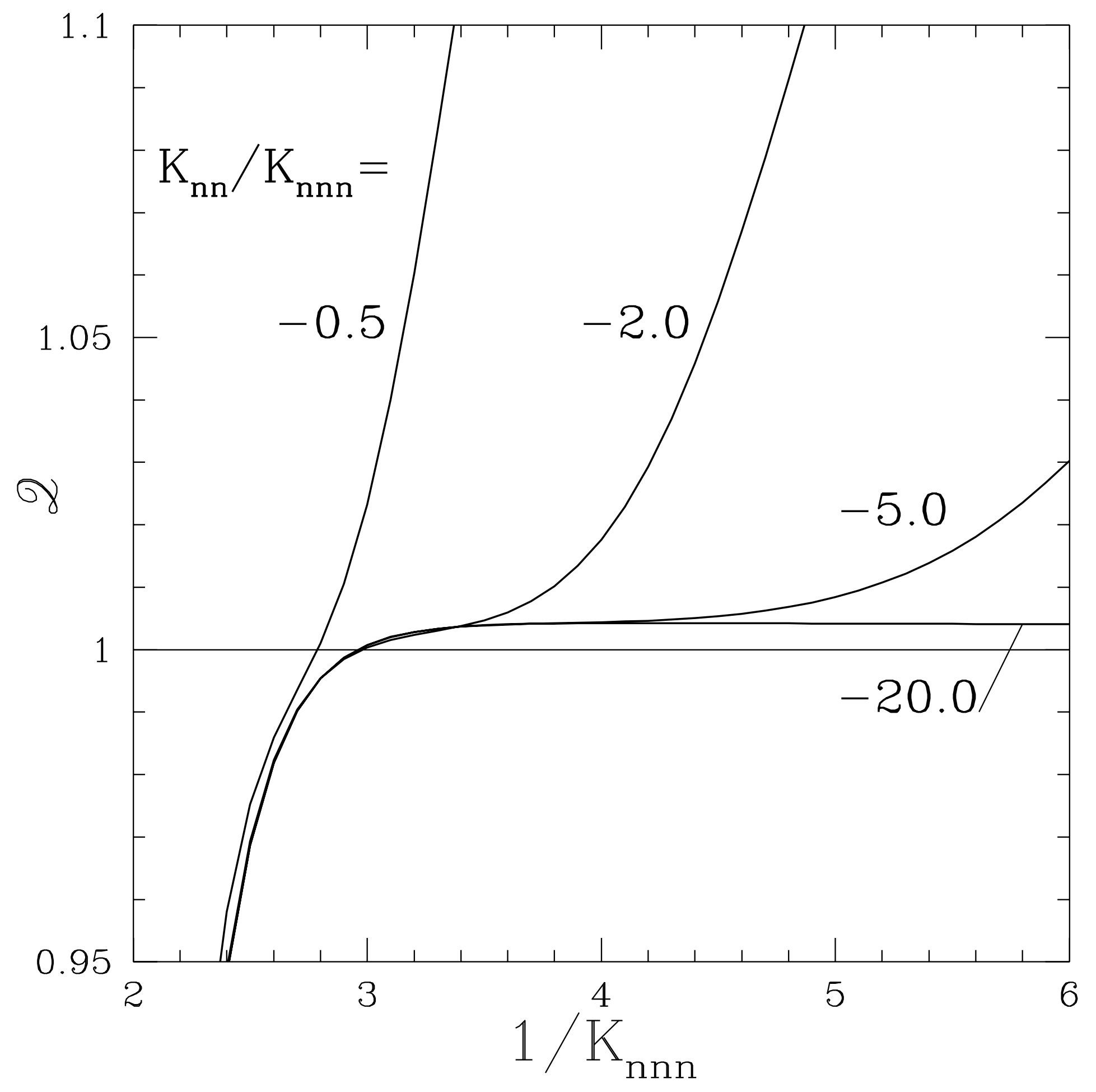




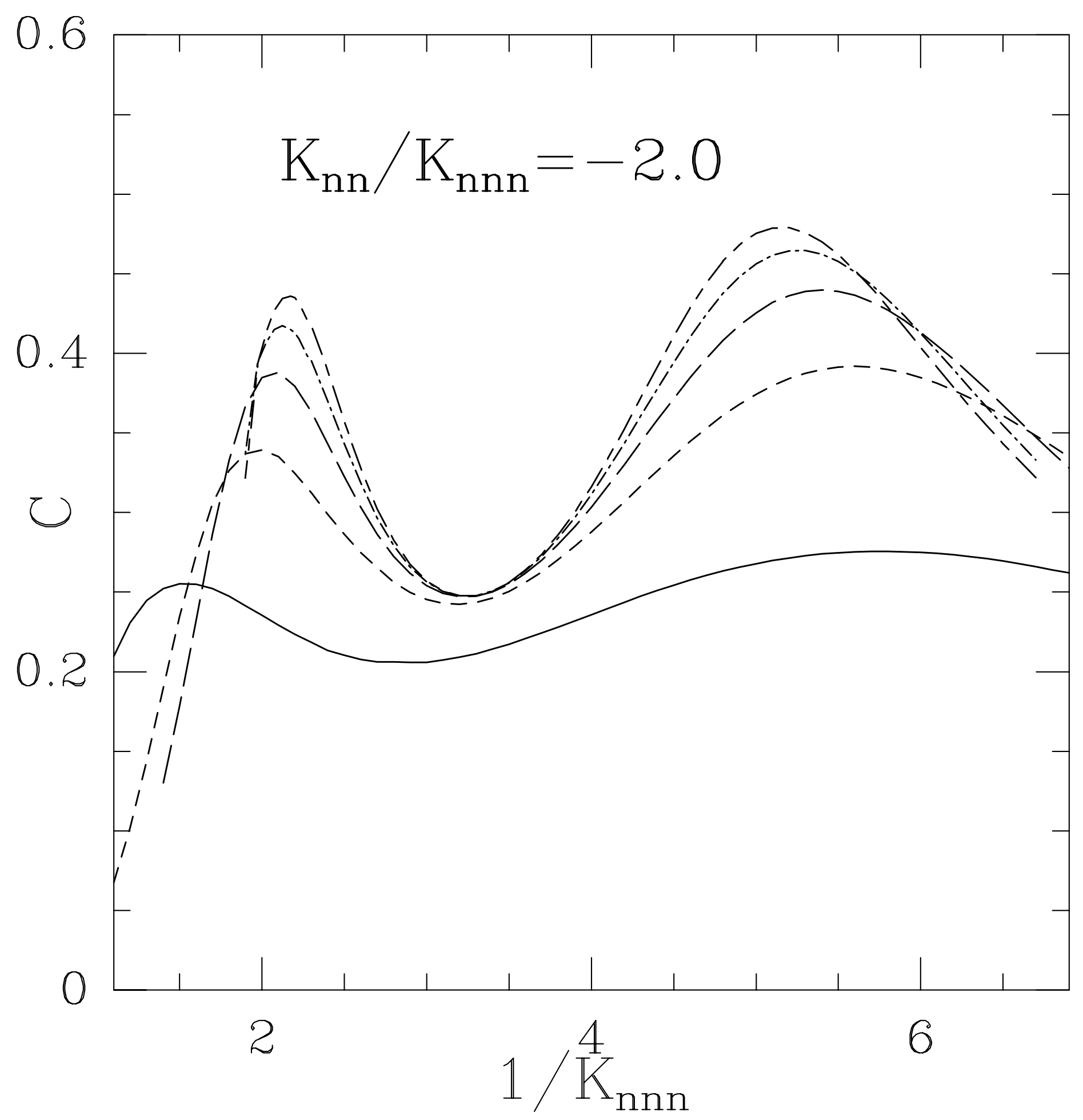




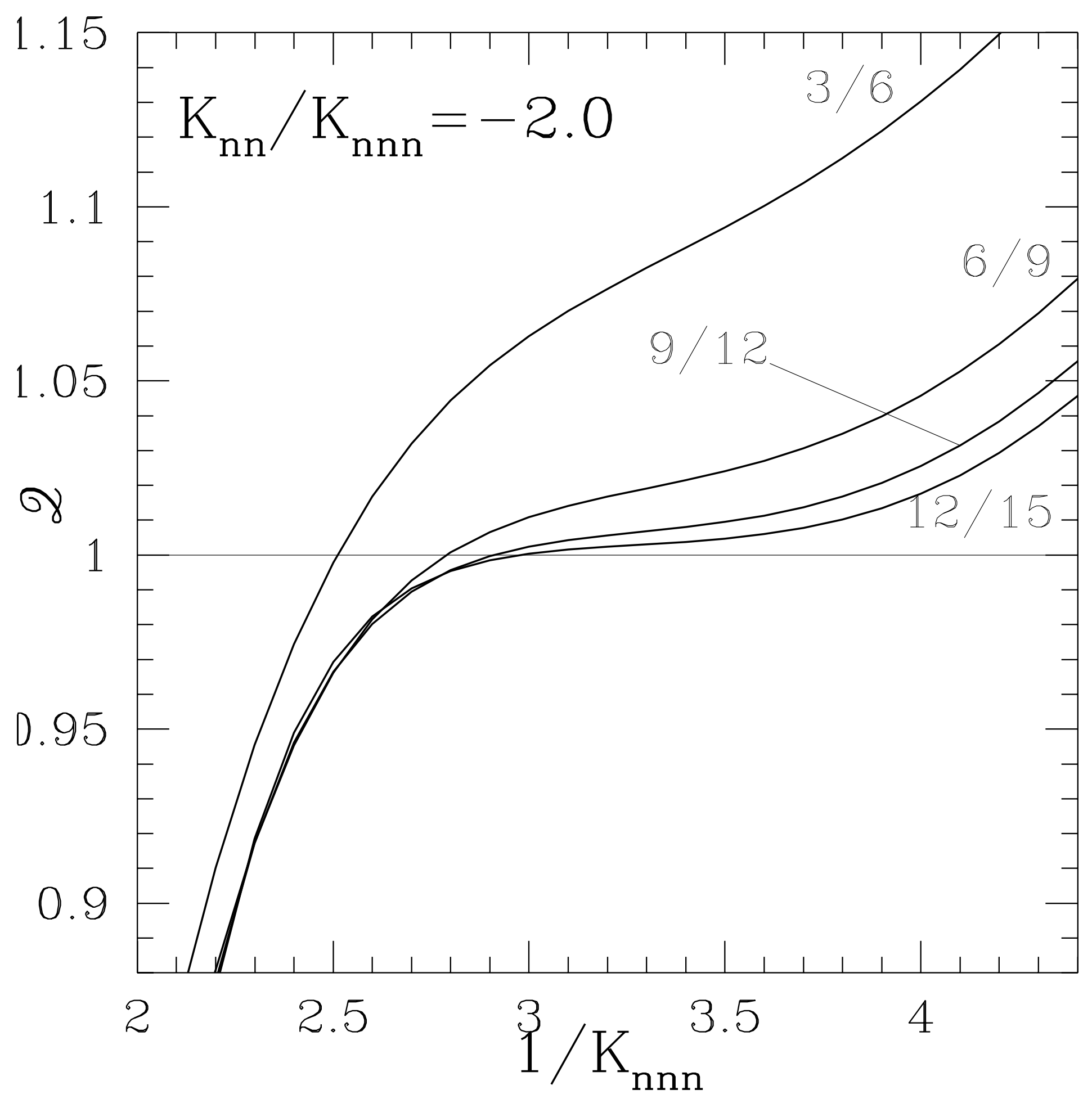




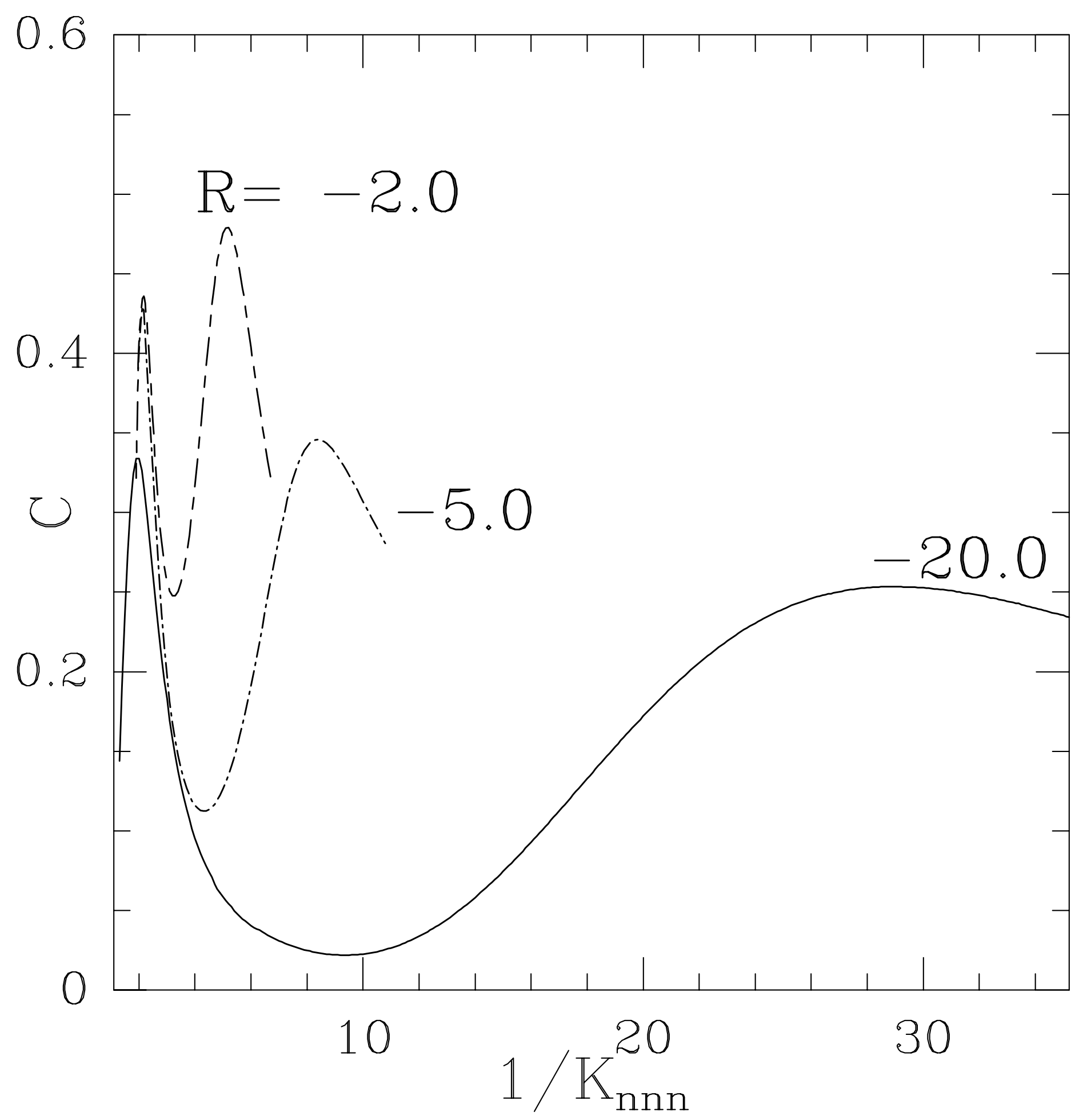




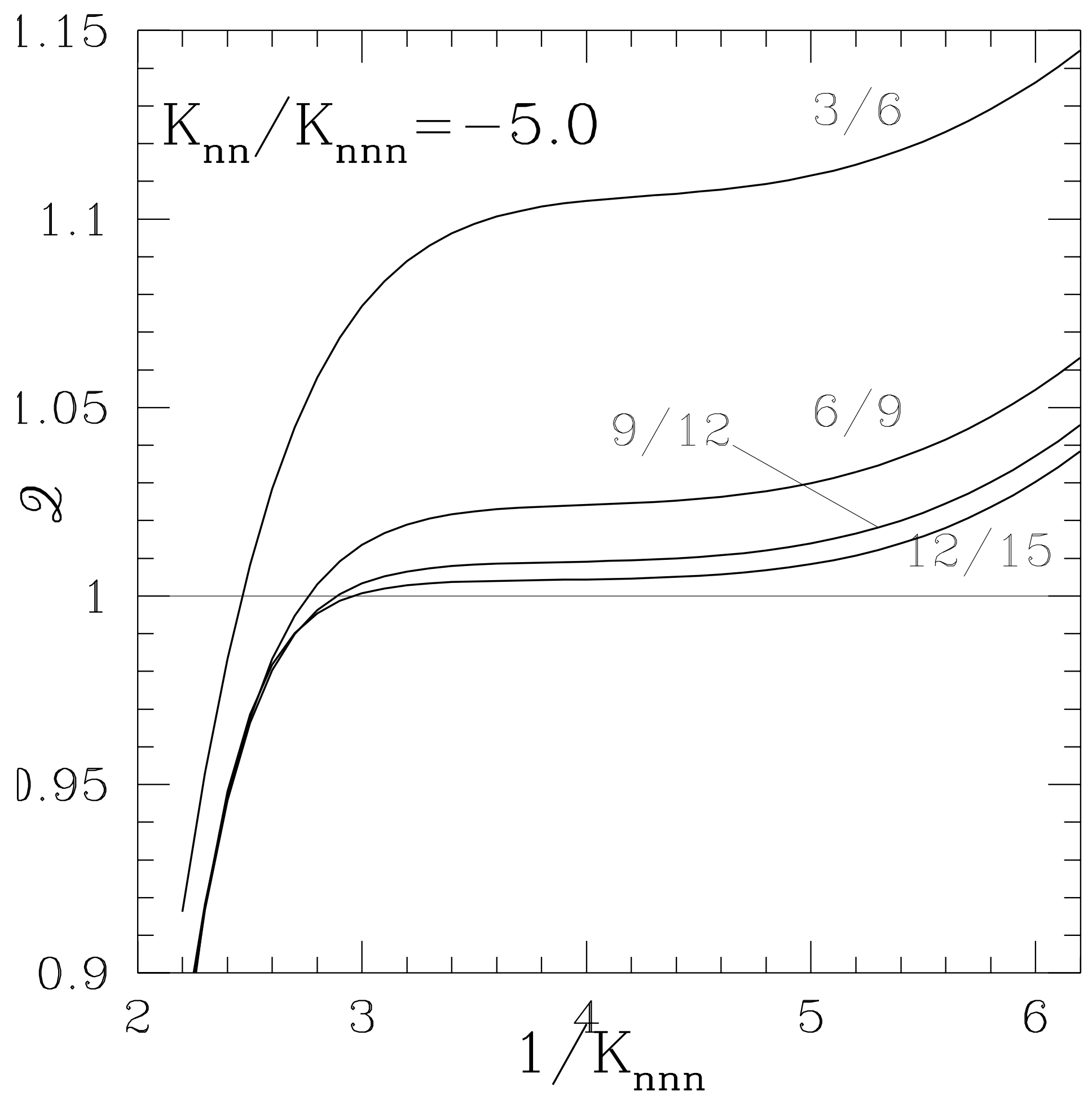

